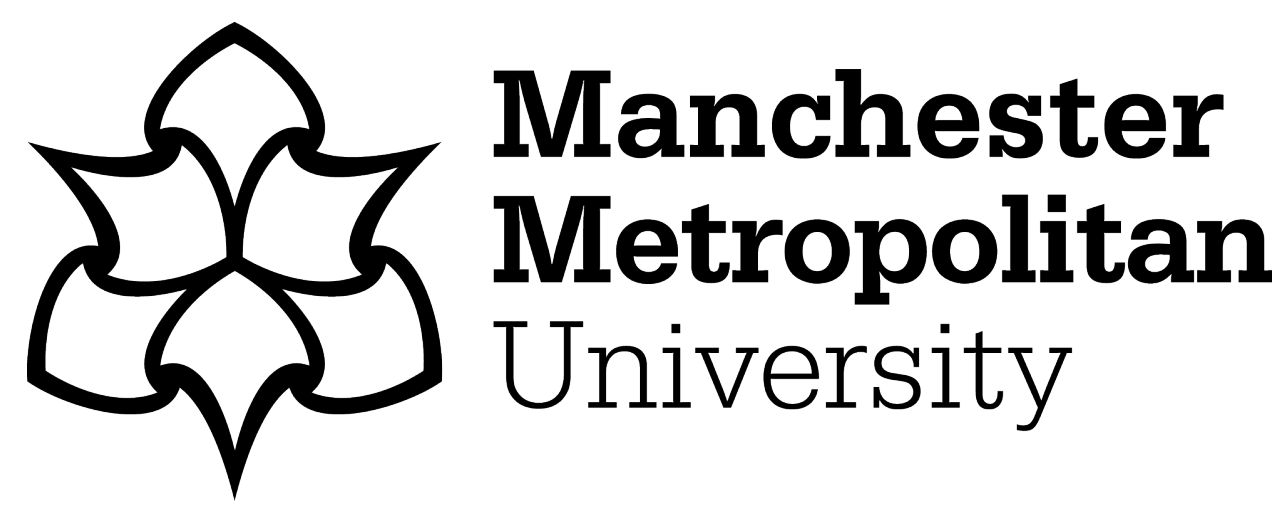

Henry, John ORCID logoORCID: https://orcid.org/0000-0003-3674-8208, Tang, Stephen, Hannenghan, Martin and Carter, Chris (2017) A Measure of Student Engagement for Serious Games and loT. In: International Conference on Technologies for E-Learning and Digital Entertainment (Edutainment 2017), Bournemouth, United Kingdom.

Downloaded from: https://e-space.mmu.ac.uk/623246/

Version: Accepted Version

Publisher: Springer

DOI: https://doi.org/10.1007/978-3-319-65849-0_28

Please cite the published version 


\title{
A Measure of Student Engagement for Serious Games and IoT
}

\author{
John Henry, Dr. Stephen Tang, Dr. Martin Hannenghan, and Dr. Chris Carter \\ Department of Computer Science, \\ Faculty of Technology and Environment, \\ Liverpool John Moores University \\ James Parsons Building, Byrom Street, Liverpool, L3 3AF, UK. \\ j.melthis@2015.1jmu.ac.uk, \{s.o.tang, \\ m.b.hanneghan, c.j.carter\}@ljmu.ac.uk \\ http://www.ljmu.ac.uk
}

\begin{abstract}
Student Engagement has been a strong topic of research for the avoidance of student drop out and the increase in grading. Serious games have highlighted benefits in engaging students, primarily through edutainment, educating via games. This article suggests a Computer Algorithm, purposed at measuring and encouraging student engagement. In addition, the algorithm accounts for sensor networks accessed both directly and through the Internet, extending its application to the Internet of Things (IoT).
\end{abstract}

\section{Introduction}

Student Engagement is a multifaceted theory that constitutes of three factors; behavioural, emotional and cognitive engagement, as described in the research review conducted by J Fredricks et al. [1]. Student engagement is usually measured through surveys based on self and/or academic self-reflection [2-6]. This paper proposes a computer algorithm that measures student engagement based on class attendance and punctuality in addition to self-perception. The calculated sum serves as data points that can be utilised in games, gamification, serious games and smart serious games.

Serious games are computer games built for non-entertainment domains, and have a presence in industries including health, advertisement, training, education, science, research, and others [7]. By harnessing the power of entertainment that gaming provides, serious games and gamification have provided a number of research and industrial solutions [8-11]. Serious Games have displayed an increase of student engagement in previous literature [12-14].

Furthermore, this paper introduces the Internet of Things (IoT) as a utility of measuring real world environmental effects on student engagement. IoT was firstly introduced by Kevin Aston [15] in 1999, aiming to identify unique objects and their virtual representations in an internet-like structure. IoT enables the connectivity of anything from any time to any place. Nowadays, researchers have expanded IoT with more technologies including sensors, network, data analysis, 
and various applications. Its applications include diverse and wide fields such as industries [16], environments [17], cities [18], transportation [19] and healthcare [20]. IoT consists of interconnected devices or Things that operate in Smart Environments and communicate data with virtual identification and/or personalities [21]. In addition, IoT accounts for an ecosystem, which is comprised of middle-ware [22], users and interconnected devices.

This paper outlines as follows; Section 2 details current research on Serious Games and the Internet of Things, in respect to the future of this research. Section 3 discusses the new terminology of Smart Serious Game, a genre of serious games that directly relates to this project. Section 4 explains the process of quantifying student engagement based on their actions in the physical world, and proposes a computer algorithm that measures, and potentially improves engagement through a game. Section 4 also presents the methodology for uncovering the proposed algorithm. Section 5 states the continuation of this research by validating the algorithm on students. Finally, the paper concludes and considers this research's limitations.

\section{Student Engagement and Serious Games}

Engagement is understood to be the involvement of people in an activity rather than the intensity of interaction [2]. Engaged students select tasks and learning objectives at the border of their comprehension and adopt a positive and optimistic approach to learning [5]. E. Skinner and M. Belmont outlined a psychological model, which drew a correlation between the competencies of students and their levels of engagement[5].

The student engagement theory is utilised for analysing dropouts in schools $[2,23]$. Research by J. Appleton et.al. outlines four subtypes of engagement: Academic, behavioural, cognitive and psychological [2]. Behaviour manipulation can be intrinsically or extrinsically triggered[1,24]. Intrinsic engagement stems from the engagement of following an instruction for the activity itself whereas extrinsic engagement relates to the desire to achieve goals and objectives related to an activity. A review conducted by J Fredricks et al., categorised student engagement into three elements: behavioural, emotional and cognitive [1]. Behavioural engagement relates to positive in-class conduct, involvement in learning relating (effort, concentration, contribution to class) and participation in activities (competitions, membership). Emotional engagement describes the feelings of students such as interest, anxiety, boredom and others. Finally, cognitive engagement focuses on the desire to complete extra curriculum activities and tasks, and the demonstration of strategic and methodical approaches to learning.

M Handlesman et al., created a questionnaire in an attempt to measure course engagement based on a four-factor dimension. The four factors comprised of skills, emotion, participation and interest, and performance. After performing validation tests against student grades they discovered correlations between engagement factors and grades, however due to the sample size acquired, their 
research could only provide an indication towards the questionnaire's effectiveness [4].

All included research into student engagement provide the psychological measures needed to monitor and evaluate engagement. All research recognises the existence of behavioural, emotional and cognitive engagement. Therefore, the algorithm and game will include these aspects of student engagement their measurements. Scientific research has proven games are engaging and immersive. In detail, research by R.Garris et.al, focuses on the instructional games and their engagement to learners to accomplish better learning outcomes [12]. Their suggested Input-Process-Output Game Model details an iterative Game Cycle that comprises of User Judgement, User Behaviour and System Feedback and encourages interacting with a game. This game cycle remains true in Smart Serious Games for student engagement.

The key difference lies in the mechanisms used to implement such an iterative cycle. User Behaviour and Judgement can now be monitored and inputted into the game through the use of network distributed sensors rather than direct input. Additionally, predictive algorithms and machine learning add another layer of information that can aid in understanding engagement from external factors, like never before.

Research into the gamification of learning experiences by A. Domnguez et al presented a solution focused on cognitive, social and emotional engagement, in an attempt to improve learning outcomes [13]. Their research provided an indication of emotional and social engagement on students; however, they noted no significant improvement on learning outcome.

Research by V. Guilln-Nieto et al., acknowledged that serious games can generate engagement and stimulation in educational environments, but focused on the effectiveness of serious games for learning [14]. Their empirical findings indicated that serious games are effective as learning tools providing that the developed solution include classroom procedures and clearly outlined learning objectives as well as game cycle and game dimensions. The structure of the serious game V. Guilln-Nieto et al., developed provides a useful insight into game elements that must be included in effective serious games for educational settings.

The core difference between the aforementioned research and this project lies in the methods of data collection and data analysis. The Student Engagement Instrument[2], and others, obtain data through questionnaires, either during classroom time, or at random intervals. This project embeds attendance as a measure, achieved through a hybrid sensor network [25], and utilises IoT to predict and inform.

The combination of IoT and Serious Games has recently been termed as Smart Serious Games (SSGs) [26]. SSGs are the merger of smart technologies, including devices and services, and the principles of Serious Games [26]. Sharma et al, detail the combination of the advantages of both technologies and its future utilisations including analytics for corporations, a tool for solving serious problems and others. 
IoT serves a pivotal role in developing predictive and reactive algorithms that measure engagement through games. The combination of a sensor network with real world data will allow correlations to be drawn. These data association allow for personalised prompting based on behaviour pattern. In detail, if a student tends to be absent on a rainy day, a personalised prompt can be created for the next time it rains to highlight that fact to the student. Prompting aside, the manipulation of data points and bonus scores can transverse the immersion of gaming into student engagement.

\section{Quantifying Student Engagement}

In the previous section, we highlighted research relating to serious games and student engagement, and identified they commonly measure engagement through qualitative feedback acquired from questionnaires. By introducing IoT, it is possible to quantify elements of student engagement, particularly behavioural. By monitoring class attendance and punctuality we receive quantitative data related to each student's engagement. Monitoring attendance and punctuality is not a new measure for student engagement, however obtaining the data through a wireless sensor network generates accurate data that has been captured in a less invasive manner. Traditional attendance monitoring systems involve pen and paper, which is susceptible to student forgery. Recent systems include RadioFrequency Identification (RFID) cards and receivers, however this is still more invasive than IoT solutions, and can disrupt class flow.

Figure 1 presents the engagement model we utilise for monitoring student engagement. Student attendance and punctuality is tracked through a wireless sensor network. Based on the time of their presence, game points are allocated for attendance and punctuality. A breakdown of the point allocation can be seen in Appendix A. Game points are also allocated through a questionnaire that is embedded into the game. The scoring system for the questionnaire follows the principles set by aforementioned research projects that utilise the same means for obtaining a measure of student engagement. In detail, a rating of 1 - 4 is given to questions that monitor distribution, effort, contribution, concentration, interest, boredom, anxiety, happiness, desire, and strategy.

The game points accumulated from sensor networks and questionnaires will allow for an aggregate scoring to be produced that can indicate a student's level of engagement. Further detail on this is provided in Section 4.

\section{Measuring Student Engagement}

This section presents the equation for calculating student engagement and the methodology behind it. The measure of student engagement will be visualised in game as a score, where a high value equates to high engagement levels.

We calculate the value by accounting for class attendance, punctuality, and the views of academics and students, gathered by game embedded questionnaires. Physical activities (attendance and punctuality), provide a static score each time 


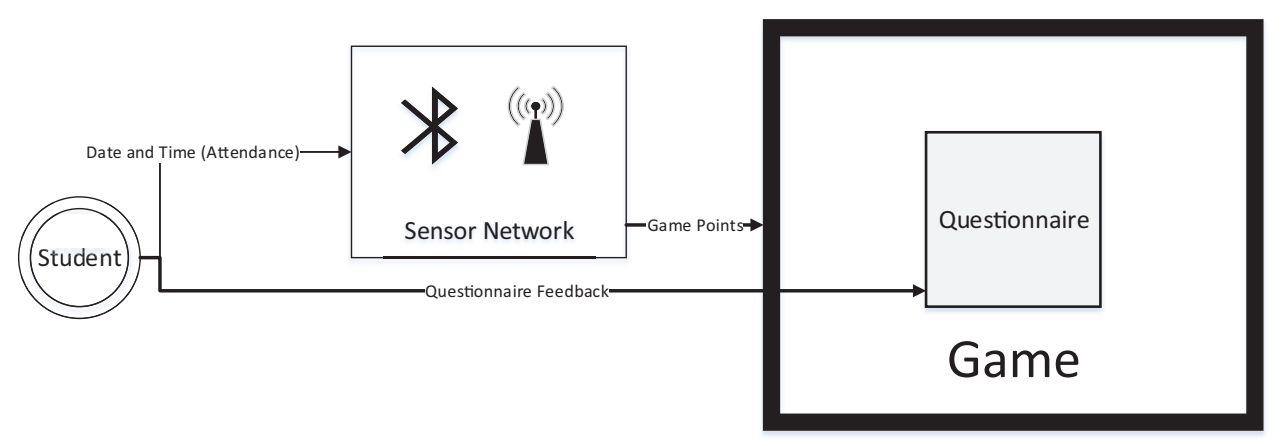

Fig. 1. An illustration of the model used for quantifying student engagement

they are completed. Students that complete a streak of game objectives will receive further rewards. For example, a student that attends all classes in a week will receive a bonus reward that directly increases the engagement score. In detail, a set game objective requires players to attend a class, this awards five points. Attending all classes in a week is a separate game objective that rewards an additional ten points if met. This method of scoring provides a points balance between those who are engaged and those who are disengaged. It is pivotal that the encouragement received in gameplay does not outscore an engaged student's score, as it can hinder the purpose of the game. Section 4.1 provides further detail on data points and the methodology used.

$$
E n=\left[\frac{1}{2}\left(C a+C p+\frac{W t}{d}\right)\right]
$$

The equation above calculates $E n$, which represents the total engagement score achieved and forms the core measurement of achievement in game. $C a$ and $C p$ are the weekly total of game points amounted by class attendance and punctuality, respectively. Wt symbolises the questionnaire weekly scores, calculated using equation 2. In detail student engagement En is formed by averaging the sum of data points achieved and the weekly total $W t$ score divided by the number of timetabled days for students. We divide by 2 as $C a$ and $C p$ require student presence for positive scoring, and is therefore considered as one factor of student engagement.

$$
W t=\left[\frac{C s}{2}+\frac{C s}{2} . .\right]
$$

$W t$ is calculated by averaging the total class score $(C s)$ from student and academic, and summing up the value of average class scores obtained through a timetabled week. Using attendance as a quantifiable measure of engagement is supported by J Frederick et al, as they deem behavioural engagement to be a crucial factor in preventing student drop-out and achieving positive academic results [1]. Referring to Figure 1, the algorithm ensures all three elements of student engagement are accounted for. Behavioural engagement will be quantified 
through presence and punctuality. A non-intrusive attendance system obtains these measurements [25]. Emotional and cognitive engagement measure through qualitative responses. Quantifying these elements would require intrusive technology, such as head scanners.

It is possible that IoT can provide an indicator of cognitive engagement. If a student chooses to attend class with severe weather conditions, high traffic congestion, or out of scheduled time, their cognitive engagement is assumed. We will conduct further research to investigate any possible correlation.

\subsection{Engagement Simulation}

We undertook a form of data simulation to identify the range of scores between engagement levels, and to determine the best equation for aggregating the total of data points. All data simulation was performed utilising spreadsheet software. To begin with, data obtained from the Web regarding traffic, weather and sunlight duration was exported into the spreadsheet. A timetable copy for a live module at Liverpool John Moores University was utilised, providing the amount of classes in a week and their respective details. At this stage three personae were developed.

Each persona represented a point in the spectrum of student engagement, therefore producing disengaged, engaged and neutral personae. We performed simulations over two hypothetical weeks. The first week utilised assumptions based on each persona. Disengaged achieves around $20 \%$ of weekly maximum $C a+C p$ and scores low on $W t$, neutral obtains $88 \%$ of $C a+C p$ with medium $W t$ score, whereas engaged is allocated with top scores.

These assumptions tested resiliently the scoring system and the equation for a balance in scores. By manipulating the points of reward through multiple iterations of calculation with the equation, a fairer score was produced and data anomalies were solved, ultimately changing the equation itself. Utilising spreadsheet software for the production of data algorithms therefore proved extremely useful as simulated data ensures a strong data algorithm is used before an application is built, reducing development time.

Simulating based on persona assumptions provided the foundation to game fairer rewards, however when taking this equation and points system to real students, further anomalies could occur. In an attempt to test this, prior to development of the game, we randomised all scores for the second week utilising embedded functionality provided by the spreadsheet software. For the second randomised week, the engaged and disengaged personae produced disengaged scores whereas neutral remained within a neutral range of score. This occurred as the attendance allocated to them by picking a random number (between 0 and 10) directly affected their respective engagement scores. An illustration of the results is located in Appendix A, where an engaged factor is symbolised with white, neutral with grey and disengaged with black.

As aforementioned, we are utilising attendance to quantify engagement, therefore the results produced indicated no preliminary issues. By utilising spread- 
sheet software and mathematical equations it is therefore possible to detect data anomalies before the development cycle begins, aiding in reducing it.

\section{$5 \quad$ Research Challenges and Future Works}

At this stage, the algorithm has been validated using data simulations of occurring and random nature. This can categorise the study as preliminary. It is important to note the focus of this paper, to propose a new algorithm for quantifying student engagement, and to document the methodology of validating a computer algorithm through data simulations, performed in spreadsheet software.

Developing the serious game and embedding the suggested algorithm ensues, allowing us to validate it with real students. We will also attempt to draw meaningful correlations between real world data and student engagement through IoT. This will provide a unique perspective on student engagement and may alter the equation's format in future, pending results from experimentation.

Post experimentation, findings will be disseminated to update on the progress of this research project. A possible fall back with our future works, students may choose to disengage with the game completely, skewering results. In this instance, participants will be questioned regarding their reasons for withdrawing.

\section{Conclusion}

Concluding, this paper introduces a computer algorithm for quantifying measuring student engagement based on an aggregate of their attendance, punctuality and subjective feedback. This data algorithm caters for Serious Games, gamification and edutainment as it utilises game points to portray results. By including IoT we acquire behavioural engagement data that we correlate against real world events. The results of this correlation aim to encourage and alert students to facts regarding their engagement. We also presented the use of data simulation for validating the data algorithm, using personae, and spreadsheet software. As aforementioned, the study is preliminary, with data from experimentation with real students needed, to validate the algorithm's effectiveness. This will be carried out as part of this research project and the findings will be disseminated accordingly.

\section{References}

1. J. A. Fredricks, P. C. Blumenfeld, and A. H. Paris: School Engagement: Potential of the Concept, State of the Evidence. Rev. Educ. Res. vol 74, pp. 59-109 (2004)

2. J. J. Appleton, S. L. Christenson, D. Kim, and A. L. Reschly: Measuring cognitive and psychological engagement: Validation of the Student Engagement Instrument, J. Sch. Psychol., vol. 44 pp. 427-445 (2006) 
3. B. D. Coller and D. J. Shernoff: Video Game-Based Education in Mechanical Engineering: A Look at Student Engagement, Int. J. Eng. Educ. vol. 25, pp. 308-317 (2009)

4. M. M. Handlesman, W. Briggs, N. Sullivan, and A. Towler: A measure of college student course engagement, J. Educ. Res. vol. 98, pp. 184-192 (2005)

5. E. A. Skinner and M. J. Belmont: Motivation in the classroom: Reciprocal effects of teacher behavior and student engagement across the school year, J. Educ. Psychol. vol. 85, pp. 571-581 (1993)

6. Brockmyer, J. H., Fox, C. M., Curtiss, K. A., McBroom, E., and Burkhart, K. M.: The development of the game engagement questionnaire: A measure of engagement in video game-playing. Journal of Experimental Social Psychology. 45, 624-634 (2009)

7. S. Tang, M. Hanneghan, and C. Carter: A platform independent game technology model for model driven serious games development, Electron. J. e-Learning. vol. 11, pp. 61-79 (2013)

8. I. Blohm and J. M. Leimeister: Gamification: Design of IT-based enhancing services for motivational support and behavioral change, Business and Information Systems Engineering. vol. 5, pp. 275-278 (2013)

9. J. W. Burke, M. D. J. McNeill, D. K. Charles, P. J. Morrow, J. H. Crosbie, and S. M. McDonough: Optimising engagement for stroke rehabilitation using serious games, Visual Computer. vol. 25, pp. 10851099 (2009)

10. C. Girard, J. Ecalle, and a. Magnan: Serious games as new educational tools: How effective are they? A meta-analysis of recent studies, Journal of Computer Assisted Learning. vol. 29, pp. 207219 (2013)

11. C. Aldrich: The complete guide to simulations and serious games :how the most valuable content will be created in the age beyond Gutenberg to Google, Pfeiffer essential resources for training and HR professionals. 533, (2009)

12. R. Garris, R. Ahlers, and J. E. Driskell: Games, Motivation, and Learning: A Research and Practice Model, Simul. Gaming. vol. 33, pp. 441-467 (2002)

13. A. Domnguez, J. Saenz-De-Navarrete, L. De-Marcos, L. Fernndez-Sanz, C. Pags, and J. J. Martnez-Herriz: Gamifying learning experiences: Practical implications and outcomes, Comput. Educ. vol. 63, pp. 380-392 (2013)

14. V. Guilln-Nieto and M. Aleson-Carbonell: Serious games and learning effectiveness: The case of Its a Deal!. Comput. Educ. vol. 58, pp. 435-448 2012.

15. K. Ashton: That 'Internet of Things' Thing, RFID Journal. vol. 22, (2009)

16. X. Jia, Q. Feng, T. Fan, and Q. Lei: RFID technology and its applications in Internet of Things (IoT), 2nd Int. Conf. on Cons. Electronics, Comm. and Networks. vol. 21, pp. 1282-1285, (2012)

17. C. Sun: Application of RFID technology for logistics on internet of things, AASRI Procedia. vol. 1, pp. 106-111, (2012)

18. A. Zanella, N. Bui, A. Castellani, L. Vangelista, and M. Zorzi: Internet of things for smart cities, Internet of Things Journal. vol. 1, pp. 22-32 (2014)

19. J. Guerrero Ibanez, S. Zeadally, and J. Contreras-Castillo: Integration challenges of intell tran. systems with connected vehicle, cloud computing, and internet of things techs., IEEE Wireless Communications. vol. 22, pp. 122-128 (2015)

20. P. Yang, M. Hanneghan, J. Qi, Z. Deng, D. Fan, and F. Dong, Improving the Validity of Lifelogging Physical Activity Measures in an Internet of Things Environment, (CIT/UCC/DASC/PICOM). (2015)

21. L. Atzori, A. Iera, and G. Morabito: The Internet of Things: A survey, Computer Networks. vol. 54, pp. 2787-2805 (2010)

22. Z. Khalid, N. Fisal, and M. Rozaini: A Survey of Middleware for Sensor and Network Virtualization, Sensors. vol. 14, pp. 24046-24097 (2014) 
23. J. D. Finn: Withdrawing from School, Rev. Educ. Res. vol. 59, pp. 117-142 (1989) 24. J. S. Eccles and A. Wigfield :Motivational Beliefs, Values, and Goals, Annu. Rev. Psychol. vol. 53, pp. 109-132 (2002)

25. J Melthis, S Tang, P Yang, et al: Topologies for combining the internet of things and serious games, Journal of Intelligent and Fuzzy Systems. 31, pp. 2685-2696 (2016) 26. D. Sharma, J. C. Lakhmi, M. Favorskaya, and R. J. Howlett: Fusion of Smart, Multimedia and Computer Gaming Technologies, Springer International Publishing. (2015)

\section{A Spreadsheet}

\begin{tabular}{|c|c|c|c|}
\hline \multicolumn{4}{|c|}{ Game Objectives (Weekly Scores) } \\
\hline \multicolumn{4}{|c|}{ Attendance (Behavioural Engagement) } \\
\hline Punctuality & Score & Classes & score \\
\hline Over 10 minutes late & 2 & Attended a class & 5 \\
\hline Between 5 and 10 minutes late & 3 & Attended all classes in a week & 10 \\
\hline Less than 5 minutes late & 5 & Attended all classes in a month & 20 \\
\hline Less than 5 minutes late in a week & 10 & Attended all classes in a semester & 40 \\
\hline $\begin{array}{l}\text { Less than } 5 \text { minutes late in a } \\
\text { month }\end{array}$ & 20 & & \\
\hline \multirow[t]{4}{*}{$\begin{array}{l}\text { Less than } 5 \text { minutes late in a } \\
\text { semester }\end{array}$} & 40 & & \\
\hline & & & \\
\hline & & WEEK 1 & \\
\hline & & Weekly Scores (Re-occuring) & \\
\hline Personas & Attendance & Questionnaire & Factor \\
\hline Engaged & 120 & 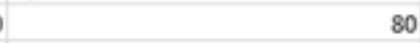 & 100 \\
\hline Neutral & 105 & 60 & 83 \\
\hline \multirow[t]{3}{*}{ Disengaged } & 24 & 10 & 17 \\
\hline & & WEEK 2 & \\
\hline & & Weekly Scores (Randomised) & \\
\hline Personas & Attendance & Questionnaire & Factor \\
\hline Engaged & 38 & 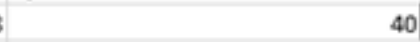 & 39 \\
\hline Neutral & 73 & 54 & 64 \\
\hline Disengaged & so & 20 & 35 \\
\hline
\end{tabular}

